\title{
Thanks to peer reviewers
}

Jian-He Xu*

Bioresources and Bioprocessing would like to express sincere gratitude to all reviewers in 2014 and 2015 for their expertise, dedication, and expeditious response.

\begin{tabular}{lll} 
Sibtain Ahmed & $\begin{array}{l}\text { Dejan Bezbradica } \\
\text { Serbia and Montenegro }\end{array}$ & $\begin{array}{l}\text { Wen-Teish Chang } \\
\text { Chinese Taiwan }\end{array}$ \\
$\begin{array}{l}\text { Joong-Hoon Ahn } \\
\text { Korea-South }\end{array}$ & $\begin{array}{l}\text { Suk Ho Bhang } \\
\text { Korea-South }\end{array}$ & $\begin{array}{l}\text { Avnish Chauhan } \\
\text { India }\end{array}$ \\
Arun Alagarsamy & Hans Blaschek & Biqiang Chen \\
India & USA & Mainland China \\
Afsheen Aman & Temitope Borokini & Bor-Yann Chen \\
Pakistan & Nigeria & Chinese Taiwan \\
Muhammad I. Amirzada & Timothy Bugg & Guo-Qiang Chen \\
Mainland China & UK & Mainland China \\
McDonald Armando & Jean-Claude Bwanganga & Hairong Chen \\
USA & Belgium & Mainland China \\
Xiyuan Bai & Menghao Cai & Hongzhang Chen \\
USA & Mainland China & Mainland China \\
V. Balasubramanian & Marli Camassola & Po Ting Chen \\
India & Brazil & Chinese Taiwan \\
Uttam Banerjee & Xuejun Cao & Qi Chen \\
India & Mainland China & Mainland China \\
Jie Bao & Sergio Capareda & Xiurong Chen \\
Mainland China & USA & Mainland China \\
Mathew Beena & Danielle Julie Carrier & Yijun Chen \\
India & USA & Mainland China \\
M. A. H. Bembenic & Sumate Chaiprapat & Keke Cheng \\
USA & Thailand & Mainland China \\
Aydin Berenjian & Chung-Soon Chang & Zhanyou Chi \\
New Zealand & Korea-South & Mainland China \\
& & \\
\hline *Correspondence: jianhexu@ecust.edu.cn & & \\
East China University of Science and Technology, Shanghai 20oz37, China & \\
\hline & & \\
\hline & &
\end{tabular}


Zhen-Ming Chi

Mainland China

Seung-Woo Cho

Korea-South

Son Chuky

Vietnam

Antonio Correia

Portugal

Otoniel Corzo

Venezuela

Maria Costa

Portugal

Muhammed Dahot

Pakistan

Chuan-Chao Dai

Mainland China

Yuyuan Dai

USA

R. F. F. de Araújo

Brazil

A. Carlos de Freitas

Brazil

Selene de Souza

Brazil

Ziwei Deng

Mainland China

Santosh Dhule

USA

Paola Didonato

Italy

Zhaoyang Ding

Mainland China

Herminia Domínguez

Spain

Qing Dong

Mainland China

Nadjib Drouiche

France

Wei Du

Mainland China

J. Singh Duhan

India
Bernd Eikmanns

Germany

Wael Eisa

Egypt

Dina El-Ghonemy

Egypt

Nahed Fakhfakh

France

Liqiang Fan

Mainland China

Yuxiang Fan

Mainland China

Swapnil Fegade

USA

Hong Feng

Mainland China

Dick Fitzgerald

Ireland

Pengcheng Fu

Mainland China

Patricia Galvez

Spain

S. Ganesan

India

Chao Gao

Mainland China

Xiaohui Gao

Mainland China

Vinod Garg

India

Jun Ge

Mainland China

Anli Geng

Singapore

Mansour Ghaffari

Iran

Younes Ghasemi

Iran

Gunjan Goel

India

Johann Görgens

South Africa
Tingyue Gu

USA

Hugh Guan

Mainland China

Yixin Guan

Mainland China

V. Gnaneswar Gude

USA

Emmanuel Guedon

France

Benjamin Gung

USA

Zheng Guo

Denmark

C. Gurramkonda

USA

Yitzhak Hadar

Israel

Yasmina Hammoui

Algeria

Chong Pik Han

Malaysia

Bingfang $\mathrm{He}$

Mainland China

Yanqing He

Mainland China

Yucai He

Mainland China

Michael Held

USA

Makoto Hibi

Japan

Ivaylo Hinkov

Bulgarie

Hirofumi Hirai

Japan

Takashi Hirasawa

Japan

Feng Hong

Mainland China

Haitong Hou

USA 
Peng Hu

Mainland China

Wei-Shou $\mathrm{Hu}$

USA

Yi Hu

Mainland China

Qiang Hua

Mainland China

Jiaofang Huang

Mainland China

Jing Huang

Mainland China

Sergio Huerta Ochoa

Mexico

Muhammad Idrees

Pakistan

Michihiko Ike

Japan

Jyoti Jadhav

India

Andre Luiz Jesus

Brazil

Huiyong Jia

Mainland China

Jianbing Jiang

USA

Min Jiang

Mainland China

Zhao Jin

Mainland China

Sang Taek Jung

Korea-South

Tae Sung Jung

Korea-South

Souichiro Kato

Japan

Christian Kennes

Spain

Zied Khiari

Canada

Jayant Khire

India
Beom Soo Kim

Korea-South

Moon Il Kim

Korea-South

Seung Wook Kim

Korea-South

Tae-Jip Kim

Korea-South

Thimo Klotzbücher

The Netherlands

Marek Kolencik

Slovakia

\section{A. Ivanov Krastanov \\ Bulgaria \\ Saravanakumar Krishnan \\ Malaysia}

Kiruba Krishnaswamy

Canada

Adepu Kumar

India

Ajay Kumar

USA

D. Senthil Kumar

India

Sachin Kumar

India

Mario Meng-Chiang Kuo

Korea-South

Buddhi P. Lamsal

USA

Hyune-Hwan Lee

Korea-South

Hanwu Lei

USA

Aitao Li

Mainland China

Fu-Sheng Li

Japan

Hui Li

Mainland China

Li Li

Mainland China
Ning Li

Mainland China

Si-Yu Li

Chinese Taiwan

Zhilin Li

USA

Zhimin Li

Mainland China

Guo-Qiang Lin

Mainland China

Chun-Ming Liu

Mainland China

Hao Liu

Mainland China

Hongjuan Liu

Mainland China

Jian-Zhong Liu

Mainland China

Jiayan Liu

Mainland China

Jinfeng Liu

Mainland China

Liming Liu

Mainland China

Luo Liu

Mainland China

Xingzhong Liu

Mainland China

Yongdong Liu

Mainland China

Zhiguo Liu

USA

Wen-Yong Lou

Mainland China

Wen-Jing Lu

Mainland China

Yanhua Lu

Mainland China

Jian Luo

Mainland China

Yane Luo

Mainland China 


\begin{tabular}{|c|c|c|}
\hline $\begin{array}{l}\text { Baodi Ma } \\
\text { Mainland China }\end{array}$ & $\begin{array}{l}\text { Bo Peng } \\
\text { UK }\end{array}$ & $\begin{array}{l}\text { Anika Scholtissek } \\
\text { Germany }\end{array}$ \\
\hline $\begin{array}{l}\text { Rocio Maceiras } \\
\text { Spain }\end{array}$ & $\begin{array}{l}\text { Kirill Peskov } \\
\text { USA }\end{array}$ & $\begin{array}{l}\text { Mohamed Shaaban } \\
\text { Germany }\end{array}$ \\
\hline $\begin{array}{l}\text { Isamu Maeda } \\
\text { Japan }\end{array}$ & $\begin{array}{l}\text { Hrvoje Petkovic } \\
\text { Slovenia }\end{array}$ & $\begin{array}{l}\text { Keelnatham Shanmugam } \\
\text { USA }\end{array}$ \\
\hline $\begin{array}{l}\text { Angel Martin } \\
\text { Spain }\end{array}$ & $\begin{array}{l}\text { Inmaculada Polo-López } \\
\text { Spain }\end{array}$ & $\begin{array}{l}\text { Sapan K. Sharma } \\
\text { India }\end{array}$ \\
\hline $\begin{array}{l}\text { Jorge Masini } \\
\text { Brazil }\end{array}$ & $\begin{array}{l}\text { Peng Qi } \\
\text { USA }\end{array}$ & $\begin{array}{l}\text { Qirong Shen } \\
\text { Mainland China }\end{array}$ \\
\hline $\begin{array}{l}\text { Daisuke Mayumi } \\
\text { Japan }\end{array}$ & $\begin{array}{l}\text { Zhigang Qian } \\
\text { Mainland China }\end{array}$ & $\begin{array}{l}\text { Yaling Shen } \\
\text { Mainland China }\end{array}$ \\
\hline $\begin{array}{l}\text { Jing Miao } \\
\text { Australia }\end{array}$ & $\begin{array}{l}\text { Yinbo Qu } \\
\text { Mainland China }\end{array}$ & $\begin{array}{l}\text { Daniel Sheng } \\
\text { Mainland China }\end{array}$ \\
\hline $\begin{array}{l}\text { Byeong Min } \\
\text { USA }\end{array}$ & $\begin{array}{l}\text { Yinbo Qureshi } \\
\text { Pakistan }\end{array}$ & $\begin{array}{l}\text { Daniel Shimizu } \\
\text { Japan }\end{array}$ \\
\hline $\begin{array}{l}\text { Ayumi Minoda } \\
\text { Japan }\end{array}$ & $\begin{array}{l}\text { K. V. Radha } \\
\text { India }\end{array}$ & $\begin{array}{l}\text { Doyun Shin } \\
\text { Korea-South }\end{array}$ \\
\hline $\begin{array}{l}\text { Karuppan Muthukumar } \\
\text { India }\end{array}$ & $\begin{array}{l}\text { Esther Ramirez-Meneses } \\
\text { Mexico }\end{array}$ & $\begin{array}{l}\text { Mitsuru Shoji } \\
\text { Japan }\end{array}$ \\
\hline $\begin{array}{l}\text { Niju Narayanan } \\
\text { USA }\end{array}$ & $\begin{array}{l}\text { L. Veeranjaneya Reddy } \\
\text { India }\end{array}$ & $\begin{array}{l}\text { Bijender Singh } \\
\text { India }\end{array}$ \\
\hline $\begin{array}{l}\text { I-Son Ng } \\
\text { Chinese Taiwan }\end{array}$ & $\begin{array}{l}\text { Guobin Ren } \\
\text { Mainland China }\end{array}$ & $\begin{array}{l}\text { Bright Singh } \\
\text { India }\end{array}$ \\
\hline $\begin{array}{l}\text { Yao Nie } \\
\text { Mainland China }\end{array}$ & $\begin{array}{l}\text { Lujing Ren } \\
\text { Mainland China }\end{array}$ & $\begin{array}{l}\text { Harikesh Bahadur Singh } \\
\text { India }\end{array}$ \\
\hline $\begin{array}{l}\text { Da-Peng Niu } \\
\text { Mainland China }\end{array}$ & $\begin{array}{l}\text { Hyeon-Su Ro } \\
\text { Korea-South }\end{array}$ & $\begin{array}{l}\text { Sarote Sirisansaneeyakul } \\
\text { Thailand }\end{array}$ \\
\hline $\begin{array}{l}\text { Renata Nowak } \\
\text { Poland }\end{array}$ & $\begin{array}{l}\text { Jayashree Rout } \\
\text { India }\end{array}$ & $\begin{array}{l}\text { Issam Smaali } \\
\text { Tunisia }\end{array}$ \\
\hline $\begin{array}{l}\text { Ernesto G. Occhiato } \\
\text { Italy }\end{array}$ & $\begin{array}{l}\text { Utpal Roy } \\
\text { India }\end{array}$ & $\begin{array}{l}\text { Ilse Smets } \\
\text { Belgium }\end{array}$ \\
\hline $\begin{array}{l}\text { Pinyakong Onruthai } \\
\text { Thailand }\end{array}$ & $\begin{array}{l}\text { Carl Safi } \\
\text { The Netherlands }\end{array}$ & $\begin{array}{l}\text { Yajun Song } \\
\text { Mainland China }\end{array}$ \\
\hline $\begin{array}{l}\text { Burhan Ozturk } \\
\text { Turkey }\end{array}$ & $\begin{array}{l}\text { Karina Salek } \\
\text { Poland }\end{array}$ & $\begin{array}{l}\text { Keerthi Srinivas } \\
\text { USA }\end{array}$ \\
\hline $\begin{array}{l}\text { Jiang Pan } \\
\text { Mainland China }\end{array}$ & $\begin{array}{l}\text { Md. Zaidul Islam Sarker } \\
\text { Malaysia }\end{array}$ & $\begin{array}{l}\text { Ajeet Kumar Srivastava } \\
\text { India }\end{array}$ \\
\hline $\begin{array}{l}\text { P. Panichayupakaranant } \\
\text { Thailand }\end{array}$ & $\begin{array}{l}\text { Gerardo C. Saucedo } \\
\text { Mexico }\end{array}$ & $\begin{array}{l}\text { Erzheng Su } \\
\text { Mainland China }\end{array}$ \\
\hline $\begin{array}{l}\text { Vasile Parvulescu } \\
\text { Romania }\end{array}$ & $\begin{array}{l}\text { Rajendra Kumar Saxena } \\
\text { India }\end{array}$ & $\begin{array}{l}\text { Haijia Su } \\
\text { Mainland China }\end{array}$ \\
\hline
\end{tabular}


Md. Mahabubur Talukder

Singapore

Jelmer Tamis

The Netherlands

Tandiono Tandiono

Singapore

Jiapeng Tang

Mainland China

Xuanping Tang

USA

Paulo Tardioli
Brazil

Miguel Teixeira

Portugal

Dirk Tischler

Germany

Si Tong

USA

Deniz Uygun

Turkey

Muthunarayanan Vasanthy India

Evgenia Vasileva-Tonkova

Bulgaria

Chokchai Wanapu

Thailand

Rufeng Wang

Mainland China

Shenqiang Wang

Mainland China

Wei Wang

Mainland China

Wei Wang

USA

Wen Wang

USA

Xiao Wang

USA

Xiaoqing Wang

USA

Yong Wang

Mainland China
Yuanpeng Wang

Mainland China

Zhilong Wang

Mainland China

Gongyuan Wei

Mainland China

Fei Wen

USA

Fai-Chu Wong

Malaysia

Haizhen Wu

Mainland China

Hui Wu

Mainland China

Jianping $\mathbf{W u}$

Mainland China

Jin Chuan Wu

Singapore

Xue-Chang Wu

Mainland China

Yishun Wu

Mainland China

Zhijun Wu

Mainland China

Zhongliu Wu

Mainland China

Jianye Xia

Mainland China

Xiaoxia Xia

Mainland China

Jingli Xie

Mainland China

Zhilong Xiu

Mainland China

Guochao Xu

Mainland China

Yi Xu

Mainland China

Zhenghong Xu

Mainland China

Zhinan Xu

Mainland China
Chen Yang

Mainland China

Jie Yang

Mainland China

Sheng Yang

Mainland China

Yang Yang

Mainland China

Kaiming Ye

USA

Qin Ye

Mainland China

Zhaoyang Ye

Mainland China

Zhaoyang Yi

Mainland China

Bingcheng Yin

Mainland China

Hanjie Ying

Mainland China

Taehyeon Yoo

Korea-South

Dinghua Yu

Mainland China

Hongwei Yu

Mainland China

Jianjia Yu

USA

Taekyung Yu

Korea-South

Changwu Yue

Mainland China

Soon-Il Yun

Korea-South

Xianhai Zeng

Mainland China

Wenbin Zhan

Mainland China

Bobo Zhang

Mainland China

Dan Zhang

Mainland China 
Jian Zhang

Mainland China

Jie Zhang

UK

Kun Zhang

Mainland China

Lin Zhang

Mainland China

Wenxue Zhang

Mainland China

Xian Zhang

USA

Xu Zhang

Mainland China

Published online: 29 March 2016
Y-H Percival Zhang

Mainland China

Zhanying Zhang

Australia

Zhijun Zhang

Mainland China

Liming Zhao

Mainland China

Xuebing Zhao

Mainland China

Yu-Hua Zhao

Mainland China
Zongbao Zhao

Mainland China

Jian-Jiang Zhong

Mainland China

Guozhong Zhou

Mainland China

Mian Zhou

Mainland China

Qi-fa Zhou

Mainland China

Christoph Zutz

Austria

Submit your manuscript to a SpringerOpen ${ }^{\odot}$ journal and benefit from:

- Convenient online submission

- Rigorous peer review

- Immediate publication on acceptance

- Open access: articles freely available online

- High visibility within the field

- Retaining the copyright to your article

Submit your next manuscript at $>$ springeropen.com 